\title{
Image-based 3D Scene Reconstruction and Rescue Simulation Framework for Railway Accidents
}

\author{
Y.Y. Nie ${ }^{1}$, Z. Tang ${ }^{* 1}$, S.H. Guo ${ }^{3}$, J.F. Yu ${ }^{1}$, Y.R. Zhu ${ }^{1}$, J. Chang ${ }^{2}$, Y. Su ${ }^{4}$, J.J. Zhang ${ }^{2}$ \\ ${ }^{1}$ State Key Laboratory of Traction Power, Southwest Jiaotong University, Chengdu, China \\ ${ }^{2}$ National Centre for Computer Animation, Bournemouth University, Poole, UK \\ ${ }^{3}$ School of software, Xiamen University, China \\ ${ }^{4}$ Sichuan Huakong Image Co., Ltd \\ E-mail:tangzhao@swjtu.edu.cn
}

\begin{abstract}
Although the railway transport is regarded as a relatively safe transportation tool, many railway accidents have still happened worldwide. In this research, an imagebased $3 D$ scene reconstruction framework was proposed to help railway accident emergency rescues. Based on the improved constrained non-linear least square optimization, the framework can automatically model the accident scene with only one panorama in a short time. We embedded the self-developed global terrain module into the commercial visualization and physics engine, which makes the commercial engine can be used to render the static scene at anywhere and simulate the dynamic rescue process respectively. In addition, a Head Mounted Device (HMD) was integrated into this framework to allow users to verify their rescue plan and review previous railway accidents in an immersive environment.
\end{abstract}

Keywords: Image-based scene reconstruction; Railway accident simulation, Virtual reality, Non-linear least square optimization, Visualization and physics engine.

\section{Introduction}

With the development of the high-speed railway network, the operating rail mileage in China has exceeded 121,000 kilometers by the end of 2015 [1], ranking first in the world. However, collision and derailment accidents in recent years have alerted governments to attach high importance to the reliability and safety of railway systems. In spite of all measures of active safety taken to avoid accidents, the statistics reveal that there is nearly one severe accident every two years [2]. Once an accident happens, a series of rescue programs are required to effectively minimize the loss of life and property. However with limited data from the on-site accident scene after an emergency occurs, it is a challenging task to draw a suitable rescue scheme.
In the recent years, computer visualization and virtual reality (VR) related three-dimensional (3D) reconstruction technologies have been applied in the railway industry [35]. However, most of existing works are concentrated on the operational inspection to ensure the railway safety[6, 7], and few works are focus on developing the methods of reconstructing the train accident scene to help program and assess the rescue plan. With applying realistic 3D visualization in building the virtual train accident scene, enormous visual information can be integrated to support the railway emergency rescue. Traditional 3D reconstruction technology includes two major methods [8]. One is LiDAR (Light detection and ranging) and the other one is airborne image photogrammetry. The LiDAR technique is mainly used to build the Digital Elevation Model (DEM) which is usually adopted to create 3D terrain. Photogrammetry is a sophisticated technique which has been applied to reconstruct various 3D models in different disciplines such as architecture [9, 10], earth sciences [11], even in forensic infography [12] and archaeometry [13]. However, the method of photogrammetry is relatively time and financial consuming. For this reason, the computer vision technology has been introduced into the photogrammetry to improve the efficiency of the 3D reconstruction, one method is so-called Image-Based Modeling (IBM). Though the performance of the IBM are less accurate comparing to laser scanner point cloud modeling, it can significantly reduce the time cost of reconstructing 3D models [14], which is critical to the railway accident rescue.

In this paper, we developed a $3 \mathrm{D}$ virtual simulation framework for train accident emergency rescue. This framework can reconstruct the train accident scene in a short time only based on one panorama, and a realistic dynamic rescue process will be presented supported by physics/game engine. This powerful and functional rescue simulation framework can be applied in programming and assessing rescue plan, physical simulation of rescue 
process, staff training, accident analysis, etc. The main contributions of this work are as follows:

(1) To help reconstruct the accident train model, we developed a panorama-CAD model based 3D modelling method for modelling the train in an accident by using the photos of the accident scene and a single carriage CAD model of the derailed train.

(2) We modeled the environmental terrain close to the accident spot using the GIS (Geographic Information System) data (elevation data and satellite images) and rendered a highly realistic accident scene in a visualization engine.

(3) We simulated the rescue process of a train accident based on the physics engine, and optimize the rescue through collision detection with surrounding obstacles distributed in an accident spot. The simulation animations can also be used for staff training after the accident.

(4) A Head Mounted Device (HMD) was integrated into this framework to allow users to verify their rescue plan and review previous railway accidents in an immersive environment.

\section{Overall framework}

The overall framework is shown in Figure 1. The 3D reconstruction algorithm of an accident train, real-world physics engine and self-developed global terrain module were integrated into this image-based framework which includes four main processes as follow:

\section{(1) Acquisition and synthesis of accident images}

It is recommended to capture the scene image of the railway accident by using a UAV (unmanned aerial vehicle). In general, it is sufficient to use only one panorama to model the accident scene. If the accident scene is too large to be included in one image, using the image mosaic algorithm to synthetic multiple pictures is a feasible solution[13].

\section{(2) 3D Reconstruction of train}

An improved constrained non-linear least square optimization algorithm was introduced to match the CAD model of the train and the involved panorama of the accident. By doing so, the framework can semiautomatically reconstruct the pose and position of the train involved in the accident scene with only one panorama in a short time.

\section{(3) Construction of accident environment}

The terrain of an accident environment was constructed by using a self-developed global terrain module. With the information of the reconstructed train and GIS data, the reconstruction result of the train accident can be rendered in the 3D visualization engine named UNIGINE to develop a 3D electronic sandbox of the train accident and display the detailed geometric information of the train accident for rescue staff.

\section{(4) Dynamics simulation of train accident rescue}

External physics models were integrated into the commercial engine (UNIGINE) which is based on rigid body dynamics to realistically simulate objects and complex interactions between them. This module reconstructs the course of the accident and provides plausible judgments of the accident causes, which are essential at the stage of rescue planning.

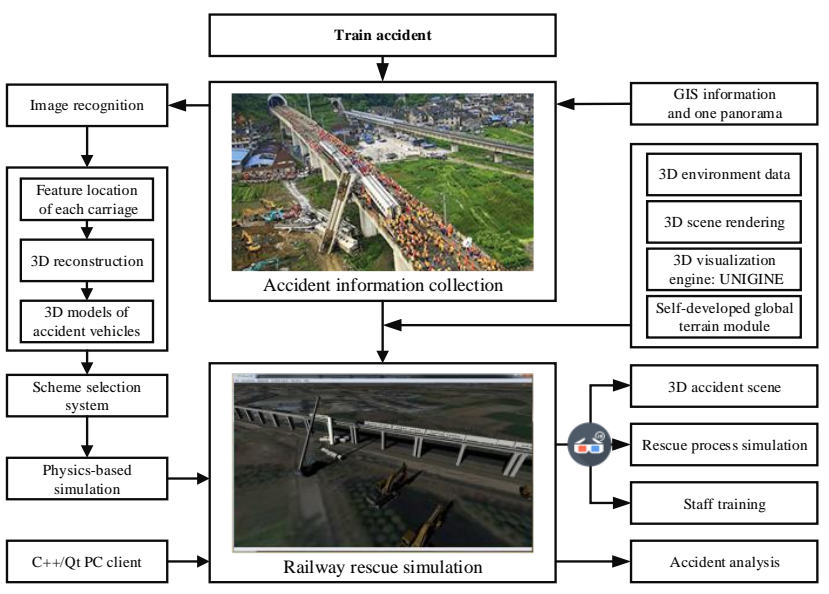

Figure 1. Overall framework

The above processes were integrated into a PC desktop software based on the $\mathrm{C}++/ \mathrm{Qt}$ framework. A highly immersive VR hardware was seamlessly integrated into this framework. Four main functions of this framework were proposed as output:

\section{(1) 3D accident scene}

Our framework outputs a highly realistic 3D scene environment which could benefit in various aspects, such as allocation of rescue resources, deployment of rescue personnel, rescue route planning, etc.

\section{(2) Rescue process simulation}

The framework is also capable of planning and simulating the rescue procedures, such as selecting rescue plans for lifting, dragging, hanging and upturning for derailed trains based on real-world physics, and eliminating possible collision and interference during the process of rescue.

\section{(3) Staff training}

A VR hardware was integrated to provide a highly immersive environment for instructor and learner of train accident rescue. In comparison with traditional training methods and tools, this immersive device could improve user experiences.

\section{(4) Accident analysis}

Based on the historical rescue process data, our framework will provide a reference for a similar train accident rescue.

The following sections will introduce the method of Image-Based Modelling to reconstruct the threedimensional position and orientation of the accident train and the method to restore the 3D terrain model of the train 
accident by using digital elevation data and HD satellite image data in Tiff format.

\section{Image-based scene modelling}

After a train accident happens, the primary task is to provide a visual description of the whole accident scene. In this section, an image-based scene modeling method was developed to reconstruct the accident scene in a high time efficiency.

\subsection{Position and pose extraction}

Before reconstructing the whole accident scene, the position and pose information about the carriages, the main body of the accident, should be recovered first. All the information required in our method is a panorama of the whole scene, which can be obtained from stitching several local photographs captured in accident spot [13].

After the panorama is acquired, considering that the traditional 3D reconstruction method is extremely timeconsuming, which focused on reconstructing the position of each pixel point about the objectives, ignoring the inherent geometry information about the main body, we introduced the CAD models of the train body to help improve the time efficiency and counteract the drawbacks of single-view based reconstruction method in reconstructing the objectives' position in depth direction. The principle of this method is shown in Figure 2.

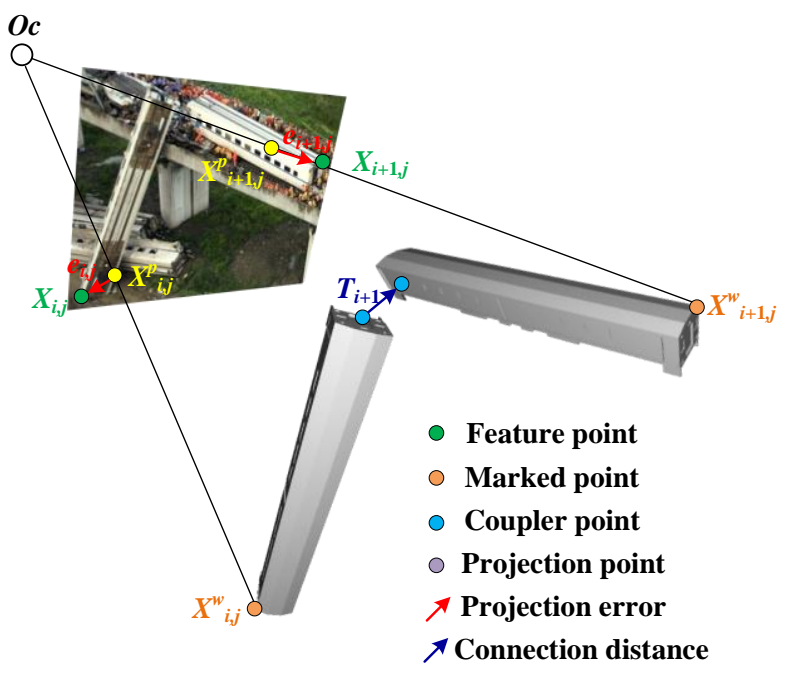

Figure 2. Extracting the pose and position of train body

In Figure 2, we manually marked several static points (the orange circles) in the CAD model of each train body and used SIFT (Scale Invariant Feature Transform) algorithm [15] to extract the feature points of each carriage (the green circles) on the panorama. In common cases, there exist errors between these static model points' projection (the yellow circles) and feature points on the image plane. Then a nonlinear least square optimization approach was adopted to adjust the position and pose of the train body to minimize these errors. To maintain the chainlike connection relationship between the couplers (the blue circles) in a train, some geometric constraints need to be added. Afterward, the optimization problem can be formulated as:

$$
\min \sum_{i=1}^{m} \frac{1}{N_{i}} \sum_{j=1}^{n_{1}}\left\|\operatorname{Proj}\left(R_{i} \cdot X_{i, j}^{w}+T_{i}\right)-X_{i, j}\right\|^{2} \cdot I_{i, j}
$$

subject to $\quad\left\|T_{i}\right\|^{2} \leq \rho_{i}, i=2, \ldots, m$,

where $m$ is the total number of carriages involved in the accident, and $X_{i, j}^{w}$ represents the 3D position of the $j$ th static point in the $i$ th carriage model in world coordinate frame (WCF). $\left[R_{i} \mid T_{i}\right]$ stand for the rotation matrix and deflection vector of the $i$ th carriage model, which are the optimization variables. It should be noticed that each rotation and deflection operation of the $i$ th model is based on the position of $(i-1)$ th model as the Figure 2 shows. From this we can know that $T_{i}$ is also the distance scalar between the $i$ th and $(i-1)$ th carriage model in WCF. Proj(.) represents the projection process from the CAD models onto the image plane. $X_{i, j}$ is the $j$ th feature point on the $i$ th carriage, and $I_{i, j}$ is denoted as the matching result of the $j$ th feature point on the $i$ th carriage by using SFIT algorithm $\left(I_{i, j}=0\right.$ or 1$) . N_{i}$ is the summation of the matching results $I_{i, j}$ in the $i$ th carriage, which has

$$
N_{i}=\sum_{j=1}^{n_{i}} I_{i, j},
$$

and $n_{i}$ is the total number of static points in the $i$ th CAD model. $\rho_{i}$ is the distance constraint between the $i$ th and $(i$ 1)th carriage model, which can display whether two adjacent carriages was separated after the train accident. After that, the trust region algorithm, a deterministic optimization approach which shows a high performance in solving constrained nonlinear least square programing [16], was applied to solve this problem (1).

After the optimization algorithm converges, the optimal pose and position information of each carriage can be obtained from $\left[R_{i} \mid T_{i}\right], i=1,2, \ldots, m$.

\subsection{Self-developed large-scale global terrain}

Considering an important fact that the railway routes have the feature of crossing many regions in the country whose geographical environments are various and complex. Once a train accident happens, the environment of the accident scene would have great influence on the rescue plan, so rendering the $3 \mathrm{D}$ environment of the accident scene is demanded as soon as possible.

To visualize the large-scale global terrain model, we developed a tool to create digital large-scale global terrain 
models by using the GIS data of digital elevation data and HD satellite image data in .Tiff format based on the techniques and algorithms for large-scale terrain visualization [17]. Figure 3 shows the process of creating large-scale global terrain model by this tool.

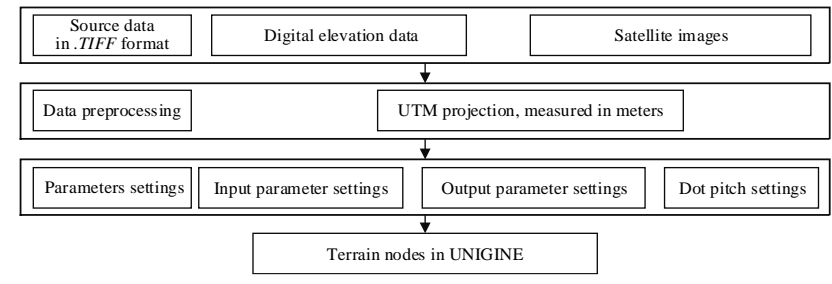

Figure 3. Flowchart of creating terrain model

The technique of LOD (Level of Detail) is integrated to efficiently display the terrain model and solve the problem of surface crack caused by LOD through increasing the number of triangular meshes of the lower level. Besides, the number of triangular meshes on flat terrain is reduced automatically, which avoids passing excessive triangular grid to the graphics so it costs less time in rendering the terrain model. The global terrain model is shown in Figure 4.

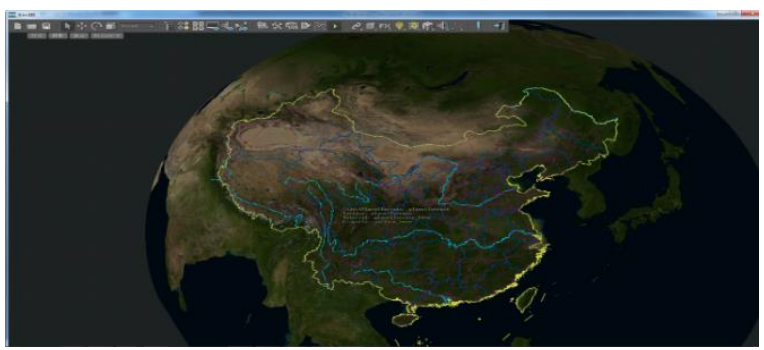

Figure 4. Global terrain model

We integrated our tool of creating large-scale global terrain model named Terrain Creator into the engine of UNIGINE by making it a plugin through the script system of UNIGINE, this work helps to reduce the time of visualizing large-scale global terrain significantly.

\subsection{Large-scale scene modelling}

In addition to the derailed train model and terrain model, there are many other fundamental models in a railway accident scene such as bridge, tracks, ballast bed, subgrade, sleeper, station, signal, tunnel, the pillar of OCS (overhead contact system)etc. All the models above are distributed along the railway routes. This paper proposed a method of large-scale scene modeling to address this issue.

According to the geometric properties of the vector information contained in the railway line, the railway line types can be divided into three classes: straight lines, circle curves and transition curves. We arranged the basic models mentioned above along the railway line by calculating the models' spatial coordinates and orientation in different railway line types. The equations of the planimetric position in dfferent railway line types are bellow:

(1) Straight lines

$$
\begin{aligned}
& x=x^{\prime}+l \sin A \\
& y=y^{\prime}+l \cos A
\end{aligned}
$$

\section{(2) Circle curves}

$$
\begin{aligned}
& x=R \sin \left(\frac{l-l_{0}}{R}+\beta_{0}\right)+m \\
& y=R\left(1-\cos \left(\frac{l-l_{0}}{R}+\beta_{0}\right)\right)+p
\end{aligned}
$$

\section{(3) Transition curves}

$$
\begin{aligned}
& x=l-\frac{l^{5}}{40 R^{2} l_{0}^{2}}+\frac{l^{9}}{3456 R^{4} l_{0}^{4}} \\
& y=\frac{l^{3}}{6 C}-\frac{l^{7}}{336 R^{3} l_{0}^{3}}+\frac{l^{11}}{42240 R^{5} l_{0}^{5}}
\end{aligned}
$$

In all these formulas, $x, y$ is the planimetric coordinates, $l$ represents the length of the railway, $A$ is azimuth, $l_{0}$ presents the length of transition curves, $\beta_{0}$ is the tangent angle, $m$ is the offset, and $p$ is the shift of the curve, $R$ represents the radius of the circular curve.

According to the equations above, we can arrange the fundamental models along a different railway line and combine them by adjusting their cohesive parts. After combining these models in the plane of railway line, we arranged these models into the terrain model by calculating their height information. The result is shown in Figure 5.

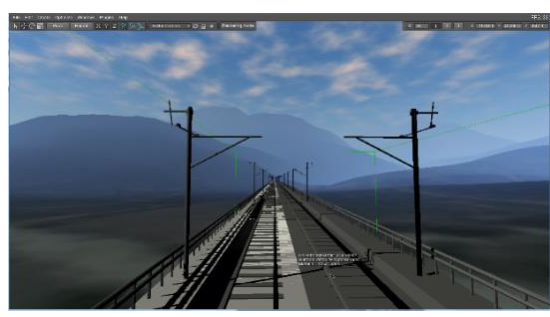

(a) Large-scale scene model

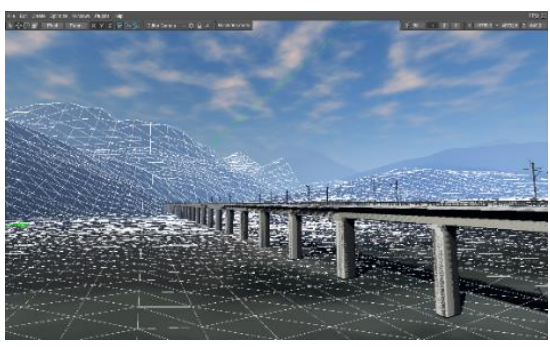

(b) Scene models arranged into the terrain

Figure 5. Large-scale scene modelling

\section{Rescue simulation}

After reconstructing the whole 3D accident scene model, we used the techniques of key-frame animations to 
combine with physics engine in UNIGINE and simulate the rescue process such as lifting, dragging, hanging and upturning for derailed trains, which can be used for staff training and education after the train accident. Once the models of a train accident are reconstructed by the modelling method mentioned above, these models are presented in the form of nodes in UNIGINE. By using the track editor in this engine, we can set the position and orientation of every node in a certain frame which is concatenated together to reproduce the process of rescue. The integration of physics engine into UNIGINE improves the realism of the simulation results of the rescue process further. A process of lifting derailed trains is shown below in Figure 6.

The railway accident scene and the simulation result of the rescue process for a train accident can be displayed by the Oculus Rift to give the rescue staffs an immersive experience. An AppOculus plugin in UNIGINE was used to support the Oculus Rift head-mounted display. This plugin provides both DirectX and OpenGL APIs so it does not require installing any additional drivers.

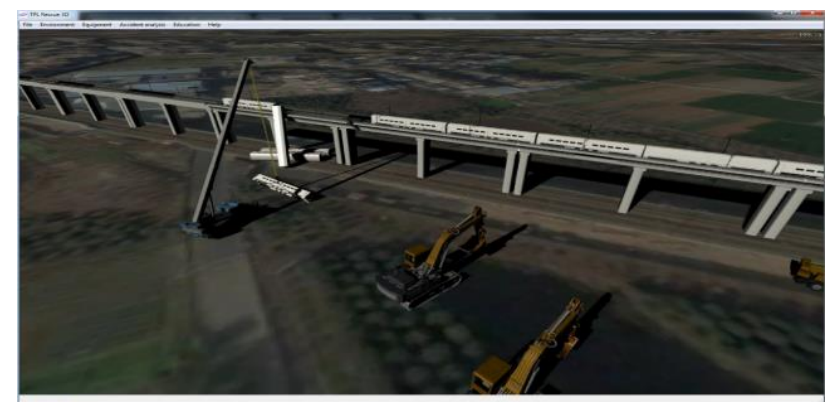

Figure 6. Simulation of lifting derailed trains

\section{Case study}

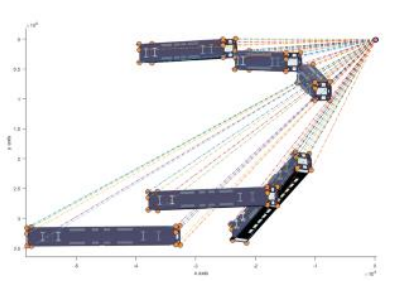

(a) Iteration: 10

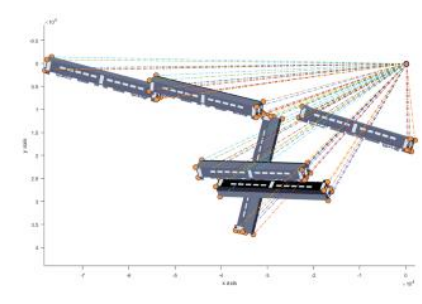

(b) Iteration: 50

Figure 8. Iteration details

\section{Conclusions and future works}

In this paper, we proposed a framework to develop an image-based railway rescue simulation framework based on a game engine. We used the photos of the accident scene to reconstruct the $3 \mathrm{D}$ train accident scene model, and

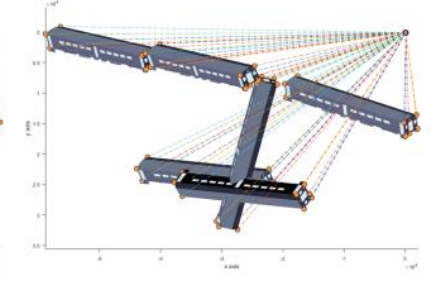

(c) Iteration: 100

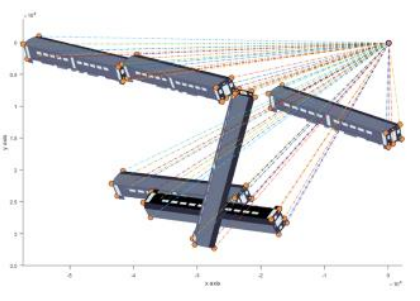

(d) Final result
In this section, a real derailment accident happened in Wenzhou, China [18] was utilized to validate the feasibility of our reconstruction and rescue simulation framework. The panorama of this accident scene is shown in Figure 7.

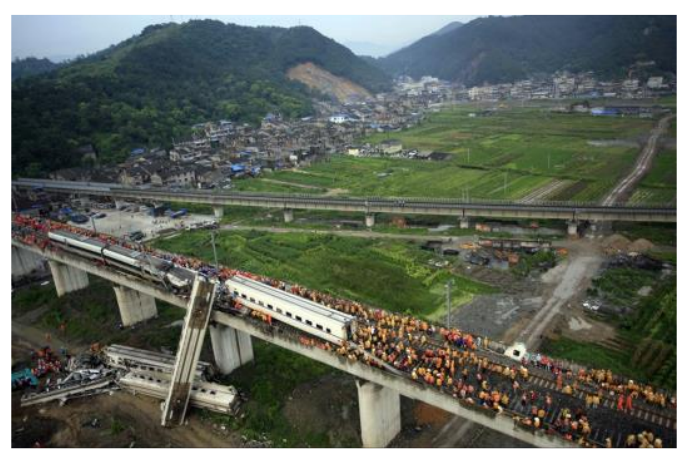

Figure 7. Derailment accident happened in Wenzhou, China

With the method described in section 3.1, the position and pose of each carriage in the scope of this panorama can be calculated (Figure 9(a)). Based on our statistics, the time cost in the process of manual intervention in matching the static points to feature points is about 310.94 seconds, and the optimization time of using trust region algorithm is about 13.03 seconds on the platform of MATLAB with Core $i 7$ processor and 8GB RAM. The total time of reconstructing the $3 \mathrm{D}$ position and pose of an entire train would last less than 8 minutes. After 493 iteration steps, the reconstruction algorithms converged and the iteration details are shown in Figure 8. Afterwards, the whole static accident scene can be recovered subsequently after importing the self-developed large-scale global terrain (Figure 9(b)). Then the electronic sandbox for the railway accident rescue simulation can be built with introducing the physics engine based key-frame animations and displayed via Oculus Rift (Figure 10).

developed a panorama-CAD model based 3D modelling method for reconstructing the train in an accident. The GIS data was used to create terrain LODs for the accident environment modelling, and a commercial visualization engine was used to render all the models in the train accident scene to offer a realistic scene model and valid information to the rescue staff. Finally, we simulated the 
railway rescue process by using the techniques of keyframe animations combined with physics engine, which benefits the rescue staff training after the train accident.

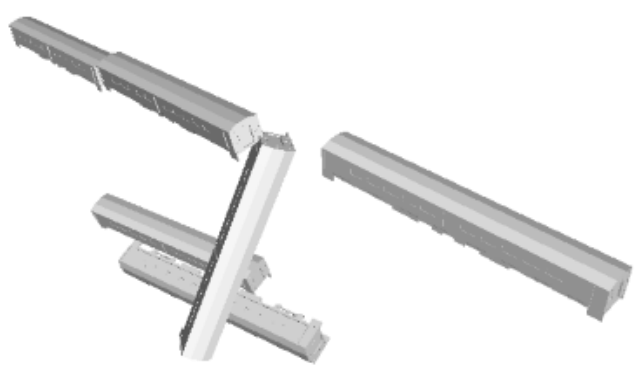

(a) Reconstructed carriages

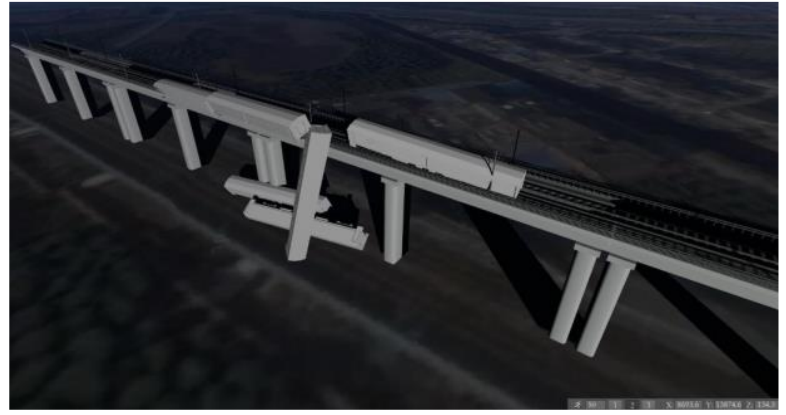

(b) Simulated accident scene

Figure 9. Reconstruction result of the accident scene
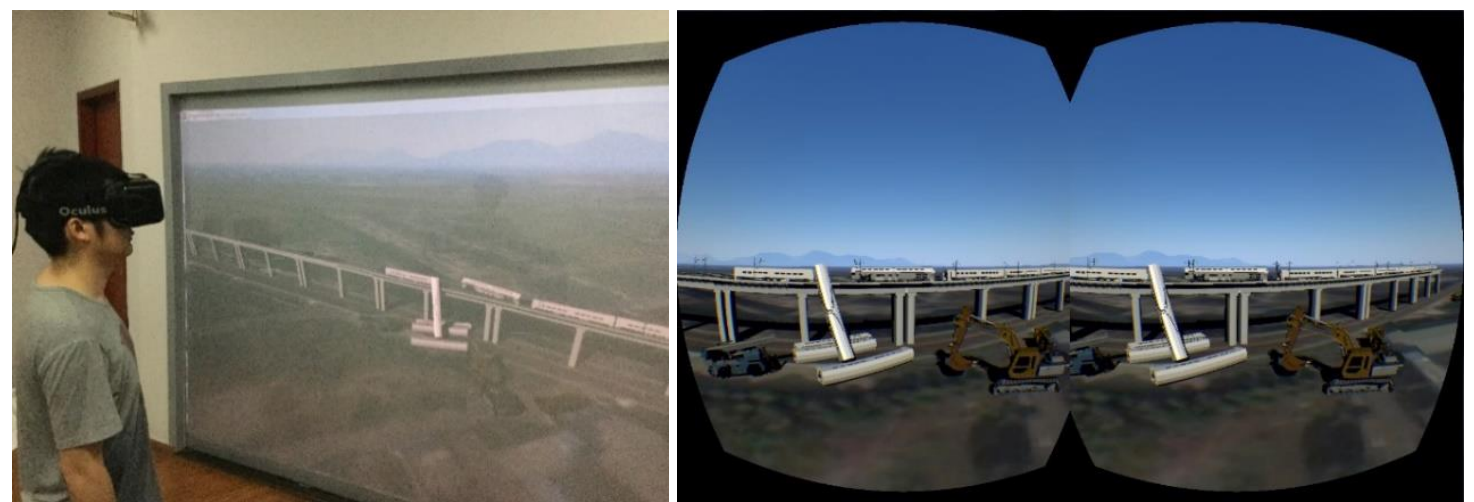

Figure 10. Visualization via Oculus Rift

Given the aforementioned advantages of the ImagedBased Modelling, reconstructing a 3D virtual railway accident scene by our framework can provide relevant information about the train accident efficiently, and help rescue staff make a reliable rescue decision effectively, which can reduce the losses caused by the train accidents. Additionally, we can create the 3D environment of the train accident rapidly by using the data of GIS, and combining the reconstruction result with the $3 \mathrm{D}$ environment models in a visualization engine to render the reconstructed $3 \mathrm{D}$ models. Therefore, an electronic sandbox for the railway rescue can be built to develop rescue plans and simulate the rescue process for staff training and accident analyzing.

In the future, there are many works to be done to improve this framework, such as reconstructing the train model by photos in rainy or foggy weather, improving the accuracy of the reconstructed scene model, establishing the database of railway accident cases to recommend reasonable rescue plans by using machine learning algorithm, etc.

\section{Acknowledgment}

This research was supported by National Natural Science Foundation of China (51405402, 51475394), the Self-developed Research Project of the State Key Lab of Traction Power (2015TPL_T06) and the Fundamental Research Funds for the Central Universities (A0920502051619-126).

\section{References}

[1] Ministry of Transport, P. R. C., Traffic and transportation industry development statistics bulletin, 2015., http://www.chinahighway.com/news/2016/1017727.php, Editor. 2016.

[2] Zarboutis, N. and Marmaras, N., "Searching efficient plans for emergency rescue through simulation: the case of a metro fire". Cognition, Technology \& Work, 2004. 6(2): p. 117-126.

[3] Lai, Y. C., et al., "Machine vision analysis of the energy efficiency of intermodal freight trains". Proceedings Of the Institution Of Mechanical Engineers Part F-Journal Of Rail And Rapid Transit, 2007. 221(3): p. 353-364.

[4] Schlake, B. W., et al., "Machine vision condition monitoring of heavy-axle load railcar structural underframe components". Proceedings of the Institution of Mechanical Engineers, Part F: Journal of Rail and Rapid Transit, 2010. 224(5): p. 499-511. 
[5] Cozens, P., et al., "Investigating personal safety at railway stations using "virtual reality" technology". Facilities, 2003. 21(7/8): p. 188-194.

[6] Aytekin, C., et al., "Railway Fastener Inspection by RealTime Machine Vision".

[7] Sawadisavi, S., Machine-vision inspection of railroad track. 2009, University of Illinois at Urbana-Champaign.

[8] Bhatla, A., et al., "Evaluation of accuracy of as-built 3D modeling from photos taken by handheld digital cameras". Automation in construction, 2012. 28: p. 116-127.

[9] Udayan, J. D., Kim, H., and Kim, J.-I., "An image-based approach to the reconstruction of ancient architectures by extracting and arranging 3D spatial components". Journal of Zhejiang University SCIENCE C, 2015. 16(1): p. 12-27.

[10] Frankl, A., et al., "Detailed recording of gully morphology in 3D through image-based modelling". Catena, 2015. 127: p. 92101.

[11] Zancajo-Blazquez, S., et al., "An Automatic Image-Based Modelling Method Applied to Forensic Infography”. PloS one, 2015. 10(3): p. e0118719.

[12] Fernández - Hernandez, J., et al., “ Image - Based Modelling from Unmanned Aerial Vehicle (UAV) Photogrammetry: An Effective, Low - Cost Tool for Archaeological Applications". Archaeometry, 2015. 57(1): p. 128-145.

[13] Brown, M. and Lowe, D. G., "Automatic panoramic image stitching using invariant features". International journal of computer vision, 2007. 74(1): p. 59-73.

[14] Golparvar-Fard, M., et al., "Evaluation of image-based modeling and laser scanning accuracy for emerging automated performance monitoring techniques". Automation in Construction, 2011. 20(8): p. 1143-1155.

[15] Lowe, D. G., "Distinctive image features from scaleinvariant keypoints". International Journal of Computer Vision, 2004. 60(2): p. 91-110.

[16] Coleman, T. F. and Hempel, C., "Computing a trust region step for a penalty function". SIAM Journal on Scientific and Statistical Computing, 1990. 11(1): p. 180-201.

[17] Döllner, J., Baumman, K., and Hinrichs, K. Texturing techniques for terrain visualization. in Proceedings of the conference on Visualization'00. 2000. IEEE Computer Society Press.

[18] Bin, C. and Guangshou, L., Aerial Photography of train accident scene in Wenzhou, http://politics.rmlt.com.cn/2011/0726/22869.shtml, Editor. 2011. 